\title{
Coherence, decoherence, and memory effects in the problems of quantum surface diffusion
}

\author{
V.V. Ignatyuk \\ Institute for Condensed Matter Physics, 1 Svientsitskii Street, 79011, Lviv, Ukraine
}

(Dated: November 8, 2018)

\begin{abstract}
We consider surface diffusion of a single particle, which performs site-to-site under-barrier hopping, fulfils intrasite motion between the ground and the first excited states within a quantum well, and interacts with surface phonons. On the basis of quantum kinetic equations for one-particle distribution functions obtained earlier we study the coherent and incoherent motion of the adparticle. In the latter case we derive the generalized diffusion coefficients and study various dynamic regimes of the adparticle. The critical values of the coupling constant $G_{c r}(T, \Omega)$, which separate domains with possible recrossing from those with the monotonic motion of the adparticle, are calculated as functions of a temperature $T$ and a vibrational frequency $\Omega$. These domains are found to coincide with the regions where the experimentally observed diffusion coefficients change its behavior from weakly dependent on $T$ to quite a sensitive function of the temperature. We also evaluate the offdiagonal (relative to the site labels) distribution functions both in the Markovian limit and when the memory effects become important. The obtained results are discussed in the context of the "long tails" problem of the generalized diffusion coefficients, the recrossing/multiple crossing phenomena, and an eventual interrelation between the adparticle dynamics at short times and the temperature dependence of the diffusion coefficients measured experimentally.

PACS numbers: 05.60.-k, 63.20.K-, 66.10.cg, 68.35.Fx
\end{abstract}

\section{INTRODUCTION}

A phenomenon of quantum diffusion of light particles (mostly, hydrogen and its isotopes), adsorbed at solid surfaces, in the last decades is a subject of joint efforts of investigators in various fields of sciences. An attention of the scientists is dictated by its relevance in the technological processes like heterogeneous catalysis [1], fuel cell production 2], chemical reactions of hydrogen transfer [3], and series of physical phenomena occurring at the fluid-gas interfaces. On the other hand, the diffusion of hydrogen is of fundamental interest from a basic physical point of view, being a favorite system for theoretical analysis [4 [6] and computer simulations 7, 8]. Wide perspectives have been opened after creation of the scanning tunnelling microscope (STM) [9], allowing to "touch" a single molecule at the surface and to carry out experiments which previously were only imagined. At the same time a rapid development of powerful methods of computer experiments like quantum molecular dynamics [10] or Monte Carlo Wave Function formalism 11] allows a direct analysis of the particle trajectories in real space and time. Recent results showing that even such the "heavy" atoms like $\mathrm{Cr}$ on the $\mathrm{Au}(111)$ surface [12] or $\mathrm{Na}$ on $\mathrm{Cu}(001)$ surfaces [13] manifest a great deal of the underbarrier tunnelling bring us to a conclusion that a traditional viewpoint on the quantum diffusion as an inherent feature of light particles only is far from reality, and a fresh look at such processes is quite topical.

A task for the theorists - to create reliable models describing a quantum surface diffusion and to develop effective methods of the calculation of diffusion coefficients with taking into account all the interactions ("adsorbateadsorbate", "substrate-adsorbate" etc.) has been ac- complished to a great extent. There should be mentioned the works 14 16] in which a bulk quantum diffusion of light particles has been studied: its description requires similar theoretical methods, and elaborated schemes can be considered as starting points for investigation of the surface quantum diffusion. They were followed by the papers where quantum hopping was moved from the bulk to the surface $4-6,17-19]$. Generally, the concept of a small polaron [4, 17, 18] or its modification [6] has been applied with going beyond the linear "adatom-phonon" coupling to consider the anharmonic terms in "adsorbate-substrate" interaction [19]. The latter case together with consideration of electronic friction in the system 14, 20] and direct "adsorbate-adsorbate" interaction 21] are very important, because they provide additional channels of particle scattering and ensure finite values of the diffusion coefficients [22].

The diffusion coefficients are traditionally determined via the Green-Kubo relations [22], the low-frequency and small wave-vector limit of the dynamic structure factor [6, 23], or zeroth moments of the "flux-flux" time correlation functions for classical 23, 24] or quantum systems [7, 8]. The latter approach is of particular interest because it allows one not only to study in detail the wellknown "recrossing/miltiple crossing" problem 7, 8], but also to derive a new quantum transition state theory [25].

The quantum diffusion coefficient is known to consist of two terms of a different physical origin. A coherent term [5, 18] characterizes the way in which the dephasing limits the band motion of the adatom by destruction of the coherence of the hopping probabilities when the adatom-thermal bath coupling induces random fluctuations of each phase. This term has a pure quantum nature and is related to the competition between the 
tunnelling mechanism, which tends to preserve the coherence, and the dephasing which characterizes damping due to the scattering process. The coherent part $D_{c o h}(T)$ of the diffusion coefficient weakly depends on temperature at low $T$ in contrast to the incoherent one $D_{\text {in }}(T)$, which decreases to zero when $T \rightarrow 0$. The incoherent contribution describes processes in which the surface dynamics induces fluctuations of the tunnelling matrix elements between two Wannier states allowing the adparticle to perform a transition from one state to another by creating or annihilating surface phonons. Though the temperature dependence of the diffusion coefficients was studied profoundly for both small polaron model [18] and its modification [6], some questions remain unclarified (especially, in the weak-coupling limit, where the contributions of $D_{c o h}(T)$ and $D_{i n}(T)$ are of the same order). The most challenging are the following: i) a justification of the multiphonon expansion; ii) a correct definition of the activation energy (e.g. when the main contribution arises from the acoustic branch of phonon spectrum); iii) an introduction of the additional channels of particles scattering ensuring correct values of the diffusion coefficients; iv) an influence of the memory effects on the temperature dependence of the diffusion coefficients and on the short-time dynamics of the adsorbate.

The latter problem should be considered from several standpoints. First of all, a thorough study of the shorttime dynamics of the adparticle allows one to distinguish between various scenarios of the adsorbate motion (a transition from the coherent to incoherent regime, a presence of multiple or long hopping etc.) that could be helpful for a deeper insight into the microscopic picture of the process [26]. Secondly, we can answer the question: what can enhance or suppress the particle motion. For instance, an eventual recrossing reduces the values of the diffusion coefficients, whereas a multiple crossing increases them. At last, such a theoretical analysis can give some recommendations for experimentalists how to perform evaluation of the diffusion coefficients more effectively. It is known [9] that at temperatures above $80 \mathrm{~K}$ the diffusion rate of hydrogen is too fast to be followed by the standard atom-tracking technique, while below $50 \mathrm{~K}$ the opposite problem occurs, and it is necessary to minimize the influence of the STM tip on the adsorbate due to a prolonged interaction. Thus, it would be tempting to relate a change in the character of the short-time dynamics of the adsorbate to possible crossover from one typical temperature behavior of the diffusion coefficients to another and to give a prognosis about the $T$-dependence of the diffusion coefficient having only an information about the adatom dynamics at the initial stage of its motion.

In the present paper, which is a logical continuation of Ref. [26], we try to give an answer to the question about the interrelation between the process of the decoherence in the "adsorbate-substrate" system (leading to the dissipative dynamics of the adsorbate) and the memory effects. Here we also use the method of the quantum kinetic equations [18, 22, 27].
The subject of our study is a single adsorbate which performs an underbarrier hopping to the nearest adsorption sites, fulfils intrasite motion between two different quantum states within a quantum well, and interacts with acoustic phonons. We define the conditions under which the adatom dynamics is definitely nonlocal in time, derive expressions for the generalized (time-dependent) diffusion coefficients, and study the influence of their long-time asymptotics on the temperature behavior of the transport coefficients measured experimentally. A particular emphasis is put on the critical regimes separating dynamics with mainly coherent contribution to the diffusion coefficient from the dominance of that of the incoherent origin. We show that a transition domain from the oscillating dynamics of the adparticle to the monotonic motion subject to the critical coupling constant $G_{c r}(T, \Omega)$ falls perfectly into the same region of $G_{c r}(T, \Omega)$ values as a crossover of the temperature dependence of the diffusion coefficients from weakly dependent on $T$ to quite a sensitive function of the temperature.

A special attention is paid to evaluation of the offdiagonal (relative to the site labels) distribution functions, which describe how fast the loss of the coherence occurs. While the study of the generalized diffusion coefficients (being related to the "velocity-velocity" autocorrelation function) allows one to make a conclusion about the recrossing phenomenon, behavior of the off-diagonal distribution functions shows us how a multiple crossing of the dividing surfaces (placed at the neighboring adsorption sites) by a moving adparticle can proceed.

Our paper is organized in the following way. In Section II we start from the unitary transformed Hamiltonian for a dissipative two-level system on a new correlated basis 17, 18, 28]. In Section III using the obtained earlier 26] non-Markovian equations for non-equilibrium distribution functions, we investigate a long-time asymptotics of the kinetic kernels determining a dissipative motion of the adsorbate. A particular case of a completely coherent dynamics, which corresponds either to $T \rightarrow 0$ or zero coupling limits, is considered. The expression for the generalized diffusion coefficients is obtained in the next Section. In Section V the Markovian approximation for these functions is considered, and a temperature behavior of the experimentally observed diffusion coefficient is studied in a weak-coupling limit. In Section VI a thorough analysis of the critical diagrams separating different kinds of the adparticle motion is performed, and the interconnection of the obtained results with those of the previous Section is established. In Section VII the off-diagonal non-equilibrium distribution function is evaluated at different values of the tunnelling constant at assumption of a continuous media; the obtained results are considered in the context of the multiple crossing, and a conclusion about validity of the Markovian approximation is inferred. In the last Section we discuss briefly the obtained results and draw final conclusions. 


\section{UNITARY TRANSFORMED HAMILTONIAN OF THE "SUBSTRATE-ADSORBATE" SYSTEM}

To specify all interactions in the "substrate-adsorbate" system we choose a Hamiltonian, which allows site-tosite tunnelling of the adsorbate, intrasite oscillations of the adparticle between the ground and the 1-st excited states within the potential well, and interaction of the adparticles with the lattice (both by density and oscillation modes). We use the same basic Hamiltonian as in Ref. [17.

Usually, in quantum diffusion problems one can consider the "substrate-adsorbate" coupling to be arbitrary. On the other hand, one-particle characteristics of the system dealt with the intersite hopping and the intrasite motion are treated as small parameters. In such a case it is useful [17, 18, 28] to start from the unitary transformed Hamiltonian on a new correlated basis, which provides a better zeroth-order representation: the sequence of unitary transformations has the effect of changing to a representation in which the adsorbate is localized at the left ( $L$-state) or at the right ( $R$-state) end of an adsorption site, and in which there is a correlated displacement of the lattice. Thus, a starting point in our study is the transformed Hamiltonian of the system of adparticles [17]:

$$
\tilde{H}=H_{\text {intra }}+H_{\text {inter }}+H_{p p}+H_{B} \equiv H^{\prime}+H_{p p}+H_{B} .
$$

The term $H_{\text {intra }}$ describes the lattice-modified intrasite dynamics of the adparticle:

$$
\begin{gathered}
H_{\text {intra }}=\sum_{s} \frac{U}{2} n_{s}\left(n_{s}-1\right)-\left(\frac{\hbar \Omega}{2} B_{s} a_{s L}^{\dagger} a_{s R}+\text { h.c. }\right), \\
B_{s}=\exp \left[-2 \sum_{q} \frac{\chi_{s q}}{\hbar \omega_{q}}\left(b_{q}-b_{q}^{\dagger}\right)\right]
\end{gathered}
$$

with a lattice induced operator exponent $B_{s}$, where denotation h.c. means Hermitian conjugation. The other denotations in Eqs. (2.2)-(2.3) are the following: $U$ means the on-site Hubbard repulsion; $\Omega$ stands for the vibrational frequency between $L$ - and $R$-states with the corresponding creation $a_{s L}^{\dagger}, a_{s R}^{\dagger}$ or annihilation $a_{s L}, a_{s R}$ operators of the adparticle at a given site $s$ (in these notations $\left.n_{s}=a_{s L}^{\dagger} a_{s L}+a_{s R}^{\dagger} a_{s R}\right)$. It has to be stressed that $\hbar \Omega$ coincides with the oscillation energy between the ground and the 1-st excited vibrational states at the assumption that zero of energy lies midway between these two levels [17, 18]. The strength $\chi_{s q}$ describes coupling of phonons with the energy $H_{B}=\sum_{q} \hbar \omega_{q} b_{q}^{\dagger} b_{q}$ to the oscillation modes of the adsorbate, and only an acoustic branch $\omega_{q}$ of the substrate motion is taken into account.

The second summand in (2.1)

$$
\begin{aligned}
& H_{\text {inter }}=\sum_{\left\langle s s^{\prime}\right\rangle} t_{c h}\left(B_{s s^{\prime}}^{L R} a_{s L}^{\dagger} a_{s^{\prime} R}+B_{s s^{\prime}}^{R L} a_{s R}^{\dagger} a_{s^{\prime} L}\right) \\
& +t_{p r}\left(B_{s s^{\prime}}^{L L} a_{s L}^{\dagger} a_{s^{\prime} L}+B_{s s^{\prime}}^{R R} a_{s R}^{\dagger} a_{s^{\prime} R}\right)
\end{aligned}
$$

is the intersite tunnelling term with end-changing (endpreserving) amplitudes $t_{c h}\left(t_{p r}\right)$, which are the linear combinations $t_{\substack{c h \\ p r}}=\frac{1}{2}\left(t_{1} \pm t_{0}\right)$ of the initial tunnelling amplitudes (usually, a condition $t_{1} \gg t_{0}$ is valid, thus an underbarrier hopping of the particle is determined by the tunnelling constant $t_{1}$ between the excited states rather than between the ground states). An abbreviation $\left\langle s s^{\prime}\right\rangle$ in (2.4) denotes a sum over the nearest-neighbor sites.

The structure of lattice induced operators $B$ in Eq. (2.4) is similar to that of (2.3):

$$
\begin{aligned}
& B_{s s^{\prime}}^{L R L}=\exp \left[-\sum_{q}\left(\Delta_{q}^{s s^{\prime}} \pm^{(+)} \delta_{q}^{s s^{\prime}}\right)\left(b_{q}-b_{q}^{\dagger}\right)\right], \\
& B_{s s^{\prime}}^{L L, R R}=\exp \left[-\sum_{q}\left(\Delta_{q}^{s s^{\prime}} \pm^{(-)} \delta_{q}^{s s^{\prime}}\right)\left(b_{q}-b_{q}^{\dagger}\right)\right], \\
& \Delta_{q}^{s s^{\prime}}=\frac{\gamma_{s q}-\gamma_{s^{\prime} q}}{\hbar \omega_{q}}, \quad{ }^{( \pm)} \delta_{q}^{s s^{\prime}}=\frac{\chi_{s q} \pm \chi_{s^{\prime} q}}{\hbar \omega_{q}},
\end{aligned}
$$

where the upper sign corresponds to the first superscript, and the strength $\gamma_{s q}$ describes coupling of phonons with the density mode of the adsorbate. In a $1 D$ case both $\gamma_{s q}$ and $\chi_{s q}$ can be expressed explicitly via the lattice parameters [17]; for a $2 D$ infinite lattice we shall introduce the lattice spectral weight functions [17, 18] to describe "substrate-adsorbate" interaction.

The last but one term of the Hamiltonian (2.1)

$$
\begin{aligned}
& H_{p p}=-\sum_{\left\langle s s^{\prime}\right\rangle}\left\{C_{s, s^{\prime}}^{D D} n_{s} n_{s^{\prime}}+2 C_{s, s^{\prime}}^{D O} n_{s}\left(n_{s^{\prime} L}-n_{s^{\prime} R}\right)\right. \\
& \left.+C_{s, s^{\prime}}^{O O}\left(n_{s L}-n_{s R}\right)\left(n_{s^{\prime} L}-n_{s^{\prime} R}\right)\right\}
\end{aligned}
$$

describes the particle-particle lattice induced interaction. For the explicit expressions of the strengths $C_{s, s^{\prime}}^{i j}$, $i, j=\{O, D\}$, and explanation of their physical meaning the reader is referred to Refs. [17, 18, 26]. It has also to be stressed that a direct "adsorbate-adsorbate" interaction can be introduced at this stage, and it will modify the expressions for $C_{s, s^{\prime}}^{i j}$ to a certain extent.

To describe the "substrate-adsorbate" interaction we consider site-independent end-changing spectral weight functions:

$$
\begin{gathered}
J(\omega)=\sum_{q} \chi_{s q}^{2} \delta\left(\omega-\omega_{q}\right), \\
J_{L R}(\omega)=\sum_{q}\left[\left(\gamma_{s q}-\gamma_{s^{\prime} q}\right)+\left(\chi_{s q}+\chi_{s^{\prime} q}\right)\right]^{2} \delta\left(\omega-\omega_{q}\right), \\
J_{R L}(\omega)=\sum_{q}\left[\left(\gamma_{s q}-\gamma_{s^{\prime} q}\right)-\left(\chi_{s q}+\chi_{s^{\prime} q}\right)\right]^{2} \delta\left(\omega-\omega_{q}\right),
\end{gathered}
$$

and end-preserving ones:

$$
\begin{aligned}
& J_{L L}(\omega)=\sum_{q}\left[\left(\gamma_{s q}-\gamma_{s^{\prime} q}\right)+\left(\chi_{s q}-\chi_{s^{\prime} q}\right)\right]^{2} \delta\left(\omega-\omega_{q}\right), \\
& J_{R R}(\omega)=\sum_{q}\left[\left(\gamma_{s q}-\gamma_{s^{\prime} q}\right)-\left(\chi_{s q}-\chi_{s^{\prime} q}\right)\right]^{2} \delta\left(\omega-\omega_{q}\right) .
\end{aligned}
$$


The function (2.7) describes the intrasite dynamics; the functions (2.8) are related to the intersite endchanging processes, while (2.9) are dealt with the intersite end-preserving processes. At low frequencies the end-changing spectral weight functions (labelled by the subscript $c$ ) are approximately given by

$$
J_{c}(\omega) \approx \begin{cases}0, & \omega<\omega_{0} \\ \eta_{c} \omega^{D-2}, & \omega>\omega_{0}\end{cases}
$$

and the end-preserving (with the subscript $p$ ) ones by

$$
J_{p}(\omega) \approx \begin{cases}0, & \omega<\omega_{0} \\ \eta_{p} \omega^{D}, & \omega>\omega_{0}\end{cases}
$$

with

$$
\eta_{c}=10 G, \quad \eta_{p}=12.5 G,
$$

given in units of the dimensionless coupling constant [17]

$$
G=\frac{\Gamma^{2}}{M \omega_{\max }^{3}} .
$$

In Eqs. (2.10)-(2.13) $D$ labels dimensionality of the lattice; $M$ denotes the mass of a substrate atom; $\omega_{\max }$ stands for the Debye frequency, and the coupling strength $\Gamma$ is expressed via the mean value of the distortion potential over the localized Wannier states.

It is seen from (2.10)-(2.11) that the lattice is allowed to possess a nonzero lowest frequency $\omega_{0}$. At first glance, the presence of a gap in the spectrum of acoustic phonons on the infinite lattice seems to be rather controversial. There were several attempts [16, 17, 29] to associate the value $\omega_{0}^{-1}$ with a finiteness of the particle life-time $\tau_{0} \gg \omega_{\text {max }}^{-1}$ in a quantum well. We are not going to repeat in our article the main reasonings of cited papers, noting that the obtained results for the surface quantum diffusion coefficients are quite insensitive to the value of $\omega_{0}$, provided the temperature is much higher than $\hbar \omega_{0} / k_{B}$ [18, 26].

Another interesting feature of the low-frequency dependence of the spectral weight functions (2.10)-(2.11) is that they are scaled like in the case of a bulk diffusion of a light inclusion, assisted by optical phonons [22, 28]. The only difference is the value of the parameter $D$, which is found to be equal to 5 .

Now let us proceed to the investigation of the adsorbate dynamics using the system of quantum kinetic equations.

\section{KINETIC EQUATIONS FOR ONE-PARTICLE NON-EQUILIBRIUM FUNCTIONS OF ADSORBATE}

The system of quantum kinetic equations for oneparticle non-equilibrium functions of the adsorbate can be obtained using the equation for the reduced density matrix $\rho_{S}(t)$ [22]. Considering the first two terms of the Hamiltonian (2.1) as a small perturbation, we can construct a closed systems of kinetic equations up to the second order in $H^{\prime}$. These equations turn out to be nonlocal in time, so it is convenient to perform a Laplace transformation for the diagonal $f_{s, s}(t)=\sum_{i=L, R}\left\langle a_{s i}^{\dagger} a_{s i}\right\rangle_{S}^{t}$ and off-diagonal $f_{s, s^{\prime}}(t)=\sum_{i=L, R}\left\langle a_{s^{\prime} i}^{\dagger} a_{s i}\right\rangle_{S}^{t}$ one-particle nonequilibrium distribution functions. In the single particle limit we obtain [26] the following chain of equations for the Laplace transforms $\tilde{f}_{s, s}(z), \tilde{f}_{s, s+n}(z)$ (the index $s+n$ means the nearest neighboring site with respect to $s)$ :

$$
\begin{aligned}
& z \tilde{f}_{s, s}(z)-f_{s, s}(t=0)=-\frac{i}{\hbar} t_{\text {inter }} \sum_{n}\left(\tilde{f}_{s, s+n}(z)-\tilde{f}_{s+n, s}(z)\right) \\
& -\tilde{\gamma}_{\text {inter }}(z)\left(2 \tilde{f}_{s, s}(z)-\sum_{n} \tilde{f}_{s+n, s+n}(z)\right), \\
& z \tilde{f}_{s, s+n}(z)-f_{s, s+n}(t=0)=-\frac{i}{\hbar} t_{i n t e r}\left(\tilde{f}_{s+n, s+n}(z)-\tilde{f}_{s, s}(z)\right. \\
& \left.+\tilde{f}_{s-n, s+n}(z)-\tilde{f}_{s, s+2 n}(z)\right) \\
& -\left(\tilde{\gamma}_{\text {inter }}(z)+\tilde{\gamma}_{\text {intra }}(z)\right) \tilde{f}_{s, s+n}(z)+\tilde{\gamma}_{L L}^{+}(z) \tilde{f}_{s+n, s}(z) .
\end{aligned}
$$

A similar chain of equations has been obtained in Ref. [18], but the authors applied the Markovian approximation for the kinetic kernels and did not study the short-time dynamics of the adsorbate.

Linearity of Eqs. (3.1)-3.2) is the result of the singleparticle approximation, and the question about statistics of the adparticle loses its significance. Note, that a similar linear approximation could be performed also at low coverage of the adsorbate. However, at low-to-moderate coverage one has to retain all nonlinear terms and at high coverage to include non-equilibrium correlation functions into the set of dynamical variables of the abbreviated description 22].

Some words have to be said about all constituents of the kinetic equations (3.1)-(3.2). The first terms of r.h.s. describe a nondissipative coherent motion of the adsorbate with the renormalized tunnelling amplitude

$$
\begin{aligned}
& t_{\text {inter }}=t_{p r} \exp \left[-\frac{1}{2} \int_{\omega_{0}}^{1} d \omega \frac{J_{p}(\omega)}{\omega^{2}} \operatorname{coth}\left(\frac{\hbar \omega}{2 k_{B} T}\right)\right] \\
& =t_{p r} \omega_{0}^{\eta_{p} k_{B} T}\left[\eta_{p} k_{B} T \sinh \left(\frac{1}{2 \eta_{p} k_{B} T}\right)\right]^{-\eta_{p} k_{B} T} \cdot
\end{aligned}
$$

In fact, $t_{\text {inter }}$ corresponds to the polaron band narrowing due to the "substrate-adsorbate" interaction. Hereafter we use dimensionless frequencies in the units of $\omega_{\max }$ and temperatures in the units of $\hbar \omega_{\max } / k_{B}$.

The kinetic kernel

$$
\tilde{\gamma}_{\text {inter }}(z)=4 \tilde{\gamma}_{L L}(z)+2 \tilde{\gamma}_{L R}(z)+2 \tilde{\gamma}_{R L}(z)
$$


corresponds to the dissipative intersite motion of the adsorbate and describes processes, when the adparticle performs series of random site-to-site hoppings (with or without the change of its quantum states) owing to the interaction with a bath. The kinetic kernel $\tilde{\gamma}_{\text {intra }}(z)$ in Eq. (3.2) describes a dissipative intrasite dynamics, when the adsorbate during its scattering from the lattice gets enough energy from the bath to be excited from the ground state to the upper level within one adsorption site (the opposite process of particle de-excitation with a phonon emission is also taken into consideration). The rates $\tilde{\gamma}_{\text {intra }}(z), \tilde{\gamma}_{\text {inter }}(z)$ can be obtained from the Laplace transformation of the kinetic kernels

$$
\begin{gathered}
\gamma_{x}(\tau)=\omega_{\max } \lambda_{x}^{2} J_{0}^{4}\left(\frac{2 t_{\text {inter }} \tau}{\hbar}\right) \\
\times \operatorname{Re}\left\{\exp \left[-\left(\varphi_{x}(0)-\varphi_{x}(\tau)\right)\right]-\exp \left[-\varphi_{x}(0)\right]\right\} \\
\gamma_{L L}^{+}(\tau)=\omega_{\max } t_{p r}^{2} J_{0}^{4}\left(\frac{2 t_{\text {inter }} \tau}{\hbar}\right) \\
\times \operatorname{Re}\left\{\exp \left[-\left(\varphi_{L L}(0)+\varphi_{L L}(\tau)\right)\right]-\exp \left[-\varphi_{L L}(0)\right]\right\}
\end{gathered}
$$

where

$\varphi_{x}(\tau)=\int_{\omega_{0}}^{1} \frac{J_{x}(\omega)}{\omega^{2}}\left[\operatorname{coth}\left(\frac{\hbar \omega}{2 k_{B} T}\right) \cos (\omega \tau)-i \sin (\omega \tau)\right]$.

In Table I we present the amplitudes $\lambda_{x}$ and the spectral weight functions $J_{x}(\omega)$, relevant to the rates (3.5)(3.6) appearing in the kinetic equations (3.1)-(3.2). The

TABLE I: Rates $\gamma_{x}$, dimensionless amplitudes $\lambda_{x}$, and spectral weight functions $J_{x}(\omega)$ along with their low-frequency limits (2.10)-2.11).

\begin{tabular}{ccc}
\hline \hline$\gamma_{x}$ & $\lambda_{x}$ & $J_{x}(\omega)$ \\
\hline$\gamma_{\text {intra }}$ & $\Omega / 2 \omega_{\max }$ & $J_{\text {intra }}(\omega)=J_{c}(\omega)$ \\
$\gamma_{L R}$ & $-\left(t_{1}+t_{0}\right) / 2 \hbar \omega_{\max }$ & $J_{L R}(\omega)=J_{c}(\omega)$ \\
$\gamma_{R L}$ & $-\left(t_{1}+t_{0}\right) / 2 \hbar \omega_{\max }$ & $J_{R L}(\omega)=J_{c}(\omega)$ \\
$\gamma_{L L}$ & $\left(t_{1}-t_{0}\right) / 2 \hbar \omega_{\max }$ & $J_{L L}(\omega)=J_{p}(\omega)$ \\
$\gamma_{R R}$ & $\left(t_{1}-t_{0}\right) / 2 \hbar \omega_{\max }$ & $J_{R R}(\omega)=J_{p}(\omega)$ \\
\hline \hline
\end{tabular}

functions (3.7) yield lattice contributions to the kinetic kernels, and the zeroth order Bessel function $J_{0}$ in (3.5)(3.6) accounts for the particle contribution. The latter function ensures a convergence of the time integrals of (3.5)-(3.6) at an arbitrary value of the coupling constant, though from the mathematical point of view it exceeds the required accuracy, being higher than of the second order in tunnelling amplitudes. This result is known to follow from going beyond the limits of the 2-nd Born approximation for the kinetic kernels [22], and provides a relaxation of the kernels to zero when $t \rightarrow \infty$. However, one has to be careful when dealing with short-time dynamics of the system, because in spite of the kinetic kernels decay the problem of energy conservation in the system appears [27]. Due to this reason we will omit the Bessel functions in the expressions (3.5)-(3.6), using instead a concept of the finite life-time $\tau_{0}=\omega_{0}^{-1}$ of the adparticle at a given adsorption cite.

A study of the long-time asymptotics of the kinetic kernels allows one to establish a one-to-one correspondence between the low-frequency behavior of the spectral weight functions $J(\omega)$ and a damping of the kernels (3.5)-(3.6) at long times. These results are summarized in Table II, where the constants $a_{i}, i=\{0, \ldots, 3\}$, are introduced just to describe a particular time behavior of $\gamma(\tau)$ (in general case, these values are defined by the system parameters). It is seen from Table II

TABLE II: Relation between a low-frequency asymptotics of the spectral weight functions and a long-time relaxation of the kinetic kernels.

\begin{tabular}{cc}
\hline \hline$J(\omega) \sim$ & $\gamma(\tau) \sim$ \\
\hline$\omega^{0}$ & $\exp \left(-a_{0} \tau^{2}\right)$ \\
$\omega^{1}$ & $\exp \left(-a_{1} \tau\right)$ \\
$\omega^{2}$ & $1 / \tau^{a_{2}}$ \\
$\omega^{n}, n \geq 3$ & $\exp \left(-a_{3}\right)$ \\
\hline \hline
\end{tabular}

that we pass from a fast relaxation of the kinetic kernels at $J(\omega) \sim \omega^{n},\{n=0,1\}$, through long tails at $n=2$ to the divergent transport coefficients (which are defined via time integrals of (3.5)-(3.6) ) at $n=3$. Note, that the system dimensionality, as it is seen from Eqs. (2.10)-(2.11), influences the long-time asymptotics of the kinetic kernels above all: in the bulk the only linear "substrate-adsorbate" coupling does not ensure the finite values of the diffusion coefficients. As it has been already mentioned, this divergency can be eliminated by introduction of the additional channels of the particle scattering (electronic friction [14, 20], anharmonic terms in "adsorbate-substrate" interaction [16, 19] or direct "adsorbate-adsorbate" interaction), which changes the low-frequency asymptotics of the spectral weight functions. For instance, taking into account the first two factors yields damping of the kinetic kernels as $\exp \left(-a_{1} t\right)$ [14, 16, 20].

Keeping in mind the data of Table II, it is possible to evaluate the long-time asymptotics of the end-changing (see first three rows of Table I) and end-preserving (last two rows) kinetic rates $\gamma_{\substack{c h \\ p r}}(\tau)$. Both rates in the strongcoupling limit $(G \geq 0.1)$ decay as Gaussian functions

$$
\begin{array}{r}
\gamma_{c}(\tau) \sim \exp \left[-\eta_{c}\left|\ln \omega_{0}\right|\left(k_{B} T \tau^{2}+i \tau\right)\right], \\
\gamma_{p}(\tau) \sim \exp \left[-\frac{\eta_{p}}{2}\left(k_{B} T \tau^{2}+i \tau\right)\right],
\end{array}
$$

while at the weak-coupling limit $(G \leq 0.01)$ the first of them still decays as a Gaussian function, but the second of them behaves as 


$$
\gamma_{p}(\tau) \sim 1 / \tau^{A \eta_{p} k_{B} T}-\omega_{0}^{2 \eta_{p} k_{B} T}, \quad A=2.15 .
$$

We will return to the expressions (3.8)-(3.10) in Section $\mathrm{V}$ when evaluating a temperature behavior of the diffusion coefficients. We also leave aside the general cases of arbitrary $n$ (both the sub-ohmic, $0 \leq n<1$, and the super-ohmic, $n>1$, regimes) in the low-frequency asymptotics of the spectral weight functions, referring readers to the review [29]. Instead, we consider a limiting case of the vanishing coupling constant $G \rightarrow 0$, or, equivalently, the low-temperature limit $T \rightarrow 0$, when the adparticle motion becomes completely coherent. For simplicity, we limit ourselves to the $1 D$, single-band $(\Omega=0)$ approximation. In such a case the kinetic equations (3.1)(3.2) can be rewritten as

$$
\dot{f}_{s, s}(t)=-\frac{i}{\hbar} t_{0} \sum_{n}\left(f_{s, s+n}(t)-f_{s+n, s}(t)\right)
$$

$\dot{f}_{s, s+n}(t)=-\frac{i}{\hbar} t_{0}\left(f_{s+n, s+n}(t)-f_{s, s}(t)+f_{s-n, s+n}(t)-f_{s, s+2 n}(t)\right)$

It is seen that in the site representation we face with a coupled chain of equations involving all the lattice labels $s$. However, this system of equations can be solved in the wave-vector representation (the details of the solution can be found in Appendix), giving the final result via the $s$-th order Bessel functions $J_{s}$ as

$$
\begin{gathered}
f_{s, s}(\tau)=J_{s}^{2}\left(2 t_{0} \hbar^{-1} \tau\right) \\
f_{s, s+1}(\tau)=\operatorname{Re}\left[f_{s, s+1}(0)\right]+i J_{s}\left(2 t_{0} \hbar^{-1} \tau\right) J_{s+1}\left(2 t_{0} \hbar\left(3{ }^{1} \cdot 1-4\right)\right.
\end{gathered}
$$

The real part of the off-diagonal distribution function can be evaluated by the methods of equilibrium statistical mechanics. It defines a strength of the transition, and does not evolve in time. Instead, the imaginary part of the $f_{s, s+n}(\tau)$ is shifted by the quarter-period with respect to the diagonal distribution functions (see Fig. 1). A particle, initially located, say, at $s=0$, starts its motion toward the nearest adsorption site $s=1$. At that time the probability to find the adparticle at the site $s=0$ reduces, the probability to meet the adparticle at the site $s=1$ increases. The imaginary part of the transition probability $f_{s, s+1}(\tau)$ reaches its maximum value when the increase/decrease of the diagonal distribution functions becomes maximal $\left(\tau^{*} \approx 1\right)$. When the particle reaches the site $s=1$ at $\tau^{*}=2$, and the value of $f_{1,1}(\tau)$ is maximal, the inverse motion of the part of the wave packet towards the site $s=0$ begins, giving the negative branch of $\operatorname{Im}\left[f_{0,1}(\tau)\right]$ at the subsequent period of time. Another part of the wave packet keeps on a motion towards the site $s=2$ yeilding a positive branch of $\operatorname{Re}\left[f_{0,2}(\tau)\right]$ and $\operatorname{Re}\left[f_{1,2}(\tau)\right]$ (not presented in Fig. 1).

In contrast to the classical picture, when a free particle performs quasi-continuous motion over the barriers,

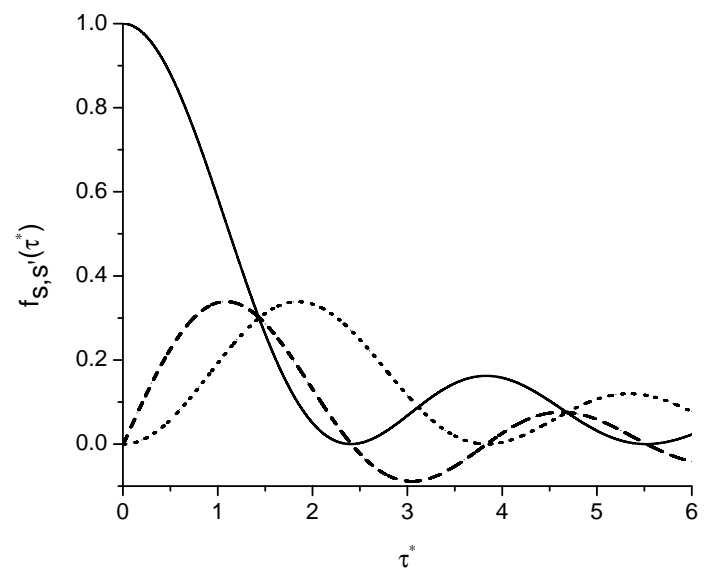

FIG. 1: The time dependence of the one-particle nonequilibrium distribution functions $f_{0,0}\left(\tau^{*}\right)$ (solid line), $f_{1,1}\left(\tau^{*}\right)$ (dotted line), and imaginary part of $f_{0,1}\left(\tau^{*}\right)$ (dashed line), given by Eqs. (3.13)-(3.14). The symbol $\tau^{*}$ denotes a time in the units $\hbar /\left(2 t_{0}\right)$.

the reason for oscillations of the distributions functions is purely quantum mechanical: a superposition of the wave packets reflection from the potential barriers and tunnelling through them.

To study the incoherent motion of adsorbate we have to obtain expressions for the generalized diffusion coefficients. This task is accomplished in the next Section.

\section{GENERALIZED DIFFUSION EQUATION FOR THE ADPARTICLE AT THE SURFACE}

Our further advance is in the manner of the generalized collective modes approach [30, 31]. To find the generalized diffusion coefficient let us solve Eq. (3.2) with respect to the hopping probabilities $\tilde{f}_{s, s+n}(z)$ and insert the obtained result into Eq. (3.1). After grouping the terms, one obtains the following equation:

$$
\begin{aligned}
& z \tilde{f}_{s, s}(z)-f_{s, s}(0)=\left\{\frac{2 t_{\text {inter }}^{2} \hbar^{-2}}{z+\tilde{\gamma}_{\text {inter }}(z)+\tilde{\gamma}_{\text {intra }}(z)+\tilde{\gamma}_{L L}^{+}(z)}\right. \\
& \left.+\tilde{\gamma}_{\text {inter }}(z)\right\}\left(\sum_{n} \tilde{f}_{s+n, s+n}(z)-2 \tilde{f}_{s, s}(z)\right) \\
& +\frac{t_{\text {inter }}^{2} \hbar^{-2}}{z+\tilde{\gamma}_{\text {inter }}(z)+\tilde{\gamma}_{\text {intra }}(z)+\tilde{\gamma}_{L L}^{+}(z)} \\
& \times \operatorname{Re}\left(2 \tilde{f}_{s-n, s+n}(z)-\tilde{f}_{s, s+2 n}(z)+\tilde{f}_{s, s-2 n}(z)\right) .
\end{aligned}
$$

The ratio in the braces describes a coherent contribution $\tilde{D}_{\text {coh }}(z)=2 t_{\text {inter }}^{2} \hbar^{-2} / \tilde{\gamma}_{\text {total }}(z)$ to the generalized diffusion coefficient and can be interpreted [18, 26] in terms of a simple model of band-type motion limited by scattering from the lattice at temperatures large relative to 
the bandwidth:

$$
\tilde{D}_{c o h}(0) \sim v^{2} / \tilde{\gamma}_{\text {total }}(0),
$$

where $v=a t_{\text {inter }} / \hbar$ denotes the average velocity of the adsorbate, $a$ stands for a substrate interatomic spacing, and

$$
\tilde{\gamma}_{\text {total }}(z)=\tilde{\gamma}_{\text {inter }}(z)+\tilde{\gamma}_{\text {intra }}(z)+\tilde{\gamma}_{L L}^{+}(z)
$$

means the total rate of scattering from the lattice. A coherent contribution $\tilde{D}_{c o h}(z)$ characterizes the way in which the dephasing limits the band motion of the adatom by destruction of the coherence of the hopping probabilities $\tilde{f}_{s, s+n}(z)$. Whereas the eigenstate of a free particle on the surface is described by a superimposition of localized Wannier states (this limiting case corresponds to the ballistic regime of motion), the coupling with the thermal bath induces random fluctuations of each phase which destroys the coherence of the state.

The second term $\tilde{D}_{i n}(z) \equiv \tilde{\gamma}_{\text {inter }}(z)$ in the braces of Eq. (4.1) is an incoherent contribution to the generalized diffusion coefficient. This is the result expected from the random walk model for diffusion with site-to-site hopping rate $\tilde{\gamma}_{\text {inter }}(z)$, describing processes of the surface phonon creation/annihilation when the particle performs a transition from one Wannier state to another.

The last term of the r.h.s of Eq. (4.1) involves the transition probabilities of the particle to perform a long hopping with $\left|s-s^{\prime}\right|>a$. In the case of consideration, when $t_{1} \ll \hbar \Omega$, this term is very small, being of the 4 -th order in tunnelling amplitude $t_{1}$. If $t_{1} \sim \hbar \Omega$, it is comparable with the second term of Eq. (4.1), being $\sim t_{1}^{2}$. Whatever the case, as it is shown in Appendix, the last term does not contribute to the overall diffusion coefficient.

Let us remind that in Section III we assumed the kinetic kernels to be independent of the site label $s$. This assumption leads to the absence of spatial non-locality in the expressions for generalized diffusion coefficients, so the memory effects only are taken into consideration. A spatial inhomogeneity is the subject of separate studies (see, e.g. Ref. [32]). The most general case of the wavevector dependent diffusion coefficients also with the time non-locality, that allows one to get a deeper insight into dynamics of the system at various time-spatial scales, is a challenging topic of non-equilibrium surface diffusion theory but lies beyond the scope of the present paper.

A multiplier $\sum_{n} \tilde{f}_{s+n, s+n}(z)-2 \tilde{f}_{s, s}(z)$ at the braces in Eq. (4.1) in the continuous media limit, when the interatomic spacing tends to zero, converts to the second derivative with respect to the space variable (for $1 D$ lattice) times $a^{2}$ or to the Laplace operator (for $2 D$ lattice in absence of the next-to-nearest-neighbor hopping) times $4 a^{2}$. It was shown in Ref. 26] that in the continuous media limit, and when coupling between the adparticle and the surface is strong enough, it is possible to obtain the Telegrapher's equation for the nonequilibrium distribution function $n(r, t)$. This equation is known to describe a correlated random walk [33] and, usually, is obtained phenomenologically by introducing special relaxation flux terms to the original diffusion equation. In 26] it was obtained rigorously by the Markovian approximation

$$
\begin{aligned}
& \tilde{D}_{\text {coh }}^{m}(z)=\frac{a^{2}}{4}\left(\frac{2 t_{\text {inter }}}{\hbar}\right)^{2} \frac{1}{z+\tilde{\gamma}_{\text {total }}(z=0)} \\
& \approx \frac{a^{2}}{4}\left(\frac{2 t_{\text {inter }}}{\hbar}\right)^{2} \frac{1}{z+\frac{1}{4} \Omega^{2} \tilde{\gamma}_{c}(z=0)}
\end{aligned}
$$

for the coherent part of the generalized diffusion coefficient and a zero-width approximation for the Gaussian functions (3.8)-3.9) (which define the incoherent term) as a consequence of different time scales for both mechanisms of dissipation.

Another remarkable feature of the short-time dynamics of the adsorbate, described by Eq. (4.1) along with approximation (4.4) for the coherent contribution to the generalized diffusion coefficient, follows from the expression for a mean square displacement of the adparticle

$$
\begin{aligned}
& \frac{\left\langle\Delta r(t)^{2}\right\rangle}{a^{2}}=\frac{2}{i \pi a^{2}} \lim _{\epsilon \rightarrow+0} \int_{\epsilon-i \infty}^{\epsilon+i \infty} \frac{\tilde{D}_{c o h}(z)+\tilde{D}_{i n}(z)}{z^{2}} \exp (z t) d z \\
& =\tilde{\gamma}_{\text {inter }}(0) t+\frac{8 t_{\text {inter }}^{2} \hbar^{-2}}{\tilde{\gamma}_{\text {total }}(0)^{2}}\left[\exp \left(-\tilde{\gamma}_{\text {total }}(0) t\right)-1+\tilde{\gamma}_{\text {total }}(0) t\right] .
\end{aligned}
$$

While in the hydrodynamic limit $t \rightarrow \infty$ Eq. (4.5) reproduces the Einstein's law for the mean square displacement

$$
\frac{\left\langle\Delta r(t)^{2}\right\rangle}{a^{2}}=\left(\tilde{\gamma}_{\text {inter }}(0)+\frac{8 t_{\text {inter }}^{2} \hbar^{-2}}{\tilde{\gamma}_{\text {total }}(0)}\right) t,
$$

in the short-time limit $t \ll 1 / \tilde{\gamma}_{\text {total }}(0)$ the second term of (4.5) converts to $4 t_{\text {inter }}^{2} \hbar^{-2} t^{2}$. The ballistic term, being obtained from the diffusion equation for $f_{s, s}(t)$, is quite uncommon because in the general case it appears only if one uses a Fokker-Planck equation for the distribution function, depending on both a coordinate and a velocity of the particle [23, 24].

At the end of this Section we would like to point your attention to the following matter. We call timedependent diffusion coefficients $D(t)$ the generalized diffusion ones, even though this denotation is usually 31, 34 attributed to the Laplace-transforms of $D(t)$. It should be stressed that the time dependence of the kinetic kernels is much more informative than frequency one: the generalized diffusion coefficient $D(t)$ is directly related to the velocity autocorrelation function $C_{s, s}^{J J}(t)$, determined on the adsorption site $s$. The investigation of its temporal behavior can help to visualize the adparticle motion both at short and long times, and it is a subject of Section VI. As for the next Section, we are going to study the temperature dependence of the diffusion coefficients, applying the Markovian approximation to $\tilde{D}_{c o h}(z)$ and $\tilde{D}_{i n}(z)$. 


\section{TEMPERATURE BEHAVIOR OF THE DIFFUSION COEFFICIENTS}

It is known that the experimentally measured values of the diffusion coefficients $D_{\text {exp }}$ are usually associated with zeroth moments of the generalized diffusion coefficients $D_{0}=\int_{0}^{\infty} D(t) d t$, which is nothing but the Markovian approximation $D_{0}=\tilde{D}(z=0)$ for their Laplace transforms. In fact, in experimental conditions one deals with evaluation of the mean square displacement of the adparticle at times much larger than $1 / \tilde{\gamma}_{\text {total }}(0)$. Thus, measuring $\left\langle\Delta r(t)^{2}\right\rangle$ one has to be sure that the influence of the transient states is excluded, and duration of the atom-tracking procedure is large enough to fall into the hydrodynamic region $t \rightarrow \infty$. Otherwise, the value $D_{\text {exp }}$ will differ from its theoretical prediction [6]. We consider the transition regimes of the adparticle motion in the next Section. In this Section we investigate the temperature behavior of the experimentally observed diffusion coefficients in the framework of two-level dissipative model.

First of all, let us determine the conditions of the validity of Markovian approximation. It is believed [6, 26] that memory effects can be neglected if the time scales describing the adsorbate motion and those of the lattice dynamics are well separated, $\omega_{\max } / \Omega \gg 1$. Stronger substrate-adsorbate coupling favors the Markovian approximation, while the weak-coupling limit usually requires consideration of the memory effects at the initial stage of the adparticle motion.

If the Markovian approximation is valid, the diffusion coefficient is determined by the Einstein's law (4.6) as

$$
\tilde{D}(0)=\frac{a^{2}}{4}\left(\tilde{\gamma}_{\text {inter }}(0)+\frac{8 t_{\text {inter }}^{2} \hbar^{-2}}{\tilde{\gamma}_{\text {total }}(0)}\right) .
$$

Taking into account the expressions (3.3) for the renormalized tunnelling amplitude and Eqs. (3.5)-(3.6), (4.3) for the kinetic kernels, and noting their time dependence (3.8)-(3.10), it is easy to perform an integration over $\tau$ and to obtain the final result.

Thus, in a strong-coupling limit $G \geq 0.1$ the diffusion coefficient is completely defined by the incoherent term:

$D_{\text {strong }}=\tilde{D}_{\text {in }}(0)=\frac{a^{2}}{\omega_{\max }}\left(\frac{t_{1}}{2 \hbar}\right)^{2} \sqrt{\frac{2 \pi}{\eta_{p}}} \frac{\exp \left(-\eta_{p} / 8 k_{B} T\right)_{5.2)}}{\sqrt{k_{B} T}}$

which, in its turn, is being determined by the endpreserving processes.

In the weak-coupling limit $G \leq 0.01$, and at a reasonable assumption $t_{1} \ll \hbar \Omega$, the diffusion coefficient

$$
D_{\text {weak }}=\frac{a^{2}}{\omega_{\max }}\left(\frac{t_{1}}{2}\right)^{2}\left[\left(\gamma_{p}+\gamma_{c}\right)+\frac{8 \exp \left(-\varphi_{p}(0)\right)}{\Omega^{2} \gamma_{c}}\right](5.3)
$$

is determined by both incoherent (the first term of Eq. (5.3) ) and coherent (the second term of Eq. (5.3)) contributions. The end-changing $\gamma_{c}$ and the end-preserving $\gamma_{p}$ rates can be presented in the following form:

$$
\begin{gathered}
\gamma_{c}=\sqrt{\frac{\pi}{\eta_{c}\left|\ln \omega_{0}\right|}} \frac{\exp \left(-\eta_{c}\left|\ln \omega_{0}\right| / 4 k_{B} T\right)}{\sqrt{k_{B} T}}, \\
\gamma_{p}=\omega_{0}^{2 \eta_{p} k_{B} T-1}\left(\frac{\omega_{0}^{(A-2) \eta_{p} k_{B} T}}{1-A \eta_{p} k_{B} T}-1\right) .
\end{gathered}
$$

It has to be noted that the expressions for $\gamma_{c}$ in both limits coincide with those of Ref. [18], while the expression for $\gamma_{p}$ in the weak-coupling limit differs from the result of cited paper, which was obtained as the multiphonon expansion of the corresponding end-preserving rates. Keeping in mind the power law behavior (3.10) of the end-preserving kernel at weak-coupling, one can justify that a multiphonon expansion is not valid in this particular case.

Now we have all necessary conditions to evaluate the temperature behavior of the surface diffusion coefficients. In Fig. 2 we present the log-log plot of the diffusion co-

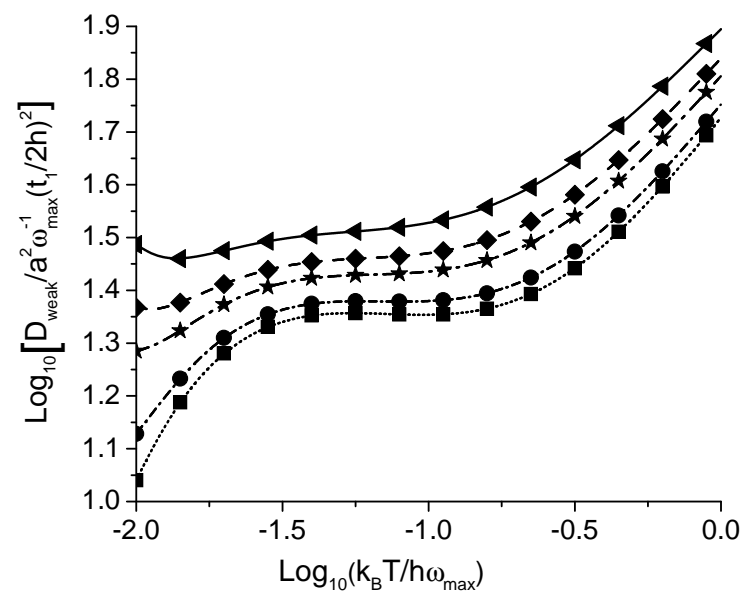

FIG. 2: Temperature dependence of the diffusion coefficient in a weak-coupling limit. Model parameters: $G=10^{-3}$, $\omega_{0} / \omega_{\max }=5 \times 10^{-4}, t_{1} / \hbar \omega_{\max }=10^{-5}$. Lines with triangles, diamonds, stars, circles and squares denote, correspondingly, the values of $\Omega / \omega_{\max }=0.2,0.25,0.3,0.5$ and 1 .

efficients in the weak-coupling limit as functions of temperature. The sum of $D_{c o h}$ and $D_{i n}$ gives rise to quite 2) a complex temperature behavior of the overall diffusion coefficient, but for all values of $\Omega$ shown, $D_{\text {weak }}$ is a relatively insensitive function of the temperature.

The arguments about validity of the Markovian approximation at values of $\Omega$, presented in Fig. 2, are not contradicting with the condition $\omega_{\max } / \Omega \gg 1$, as long as we are not interested in the adsorbate motion at short time scales: according to Eq. (4.4), the zeroth moment of $D_{\text {coh }}(t)$ can be calculated at any value of $\Omega$. We will see in the next Section that it is not true if one investigates the intermediate regimes $t \sim 1 / \tilde{\gamma}_{\text {total }}(0)$, and it is necessary to consider a diffusion equation which is nol-local in time. 
In Fig. 3 we show the plot of the full diffusion coefficient at stronger coupling to the lattice but still in the weak-coupling regime.

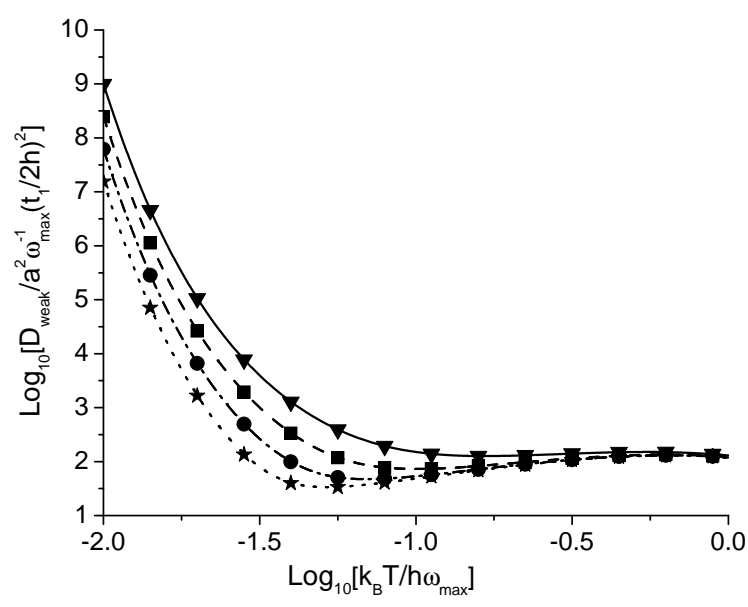

FIG. 3: Temperature dependence of the diffusion coefficient in a weak-coupling limit. Model parameters: $G=10^{-2}$, $\omega_{0} / \omega_{\max }=5 \times 10^{-4}, t_{1} / \hbar \omega_{\max }=10^{-5}$. Lines with triangles, squares, circles and stars denote, correspondingly, the values of $\Omega / \omega_{\max }=0.25,0.5,1$, and 2 .

The temperature dependence is quite different from that shown in Fig. 2: at low temperatures the diffusion is dominated by the coherent contribution, which is a strongly decreasing function of $T$. According to (5.3)-(5.4), the coherent contribution to the diffusion coefficient behaves as $\exp \left(E_{c} / k_{B} T\right)$ at low temperatures and as $\sqrt{k_{B} T}$ at higher temperatures. At high temperatures the main contribution comes from the incoherent term, and $D_{\text {weak }}$ is a slowly increasing function of $T$.

Let us briefly discuss the temperature behavior of $D_{\text {weak }}$ at low temperatures, shown in Fig. 3. A qualitatively similar increase of the diffusion rate of $\mathrm{H}$ on $\mathrm{Cu}(001)$ below $20 \mathrm{~K}$ was observed experimentally in Ref. [9]. However, in our case this behavior is just a result of the used two-level dissipative model, whereas in Ref. [9] it was attributed to the change of nonadiabatic response of the thermally excited electron-hole pairs to the diffusing particle. When plotting Figs. 2 and 3 we just emphasize that a crossover from one kind of temperature dependence of the diffusion coefficient to another takes place even in a relatively simple model. In the next Section we will show that this crossover coincides perfectly (regarding to the coupling constant $G$ ) with the change of the character of adparticle dynamics at short times, when the memory effects have to be taken into account.

\section{TRANSITION REGIMES OF THE GENERALIZED DIFFUSION COEFFICIENTS}

We have already mentioned that for visualization of the processes of adparticle motion at short and intermediate times it is much more convenient to perform an inverse Laplace transform of the generalized diffusion coefficients according to

$$
\begin{aligned}
& D_{\text {coh }}(t)=\operatorname{Re}\left[\frac{\left(a t_{\text {inter }}\right)^{2}}{2 \pi i \hbar^{2}} \lim _{\epsilon \rightarrow 0} \int_{\epsilon-i \infty}^{\epsilon+i \infty} d z \exp (z t) \frac{1}{z+\tilde{\gamma}_{\text {total }}(z)}\right] \\
& =\left(\frac{a t_{\text {inter }}}{\hbar}\right)^{2} \operatorname{Re}\left[\sum_{i=1}^{\infty} \exp \left(-z_{i} t\right) \frac{1}{1+\tilde{\gamma}_{\text {total }}^{\prime}\left(z_{i}\right)}\right]
\end{aligned}
$$

The summation in (6.1) in accordance with the residue theorem runs over all poles $z_{i}$ of the integrand, which obey the condition $\operatorname{Re}\left[z_{i}\right] \leq 0$. The expression (6.1) resembles the results of the generalized collective modes theory [30, 31], postulating the additive contribution of each collective excitation to a particular time correlation function. In our case, the summation is extended to the infinite number of poles, and a main contribution comes from the terms with maximal values of $\operatorname{Re}\left[z_{i}\right]$ and weight factors $\left[1+\tilde{\gamma}_{\text {total }}^{\prime}\left(z_{i}\right)\right]^{-1}$. It has also to be mentioned that the expression for $D_{\text {coh }}(t)$ can be even more complicated if one deals with poles of the order $n>1$.

The expression for $D_{i n}(t)$ follows immediately from the definition (3.4) of the kernel $\tilde{\gamma}_{\text {inter }}(z)$ and can be written down via the end-changing/end-preserving functions (3.8)-(3.10) as

$$
D_{i n}(t)=\left(a t_{1} / h\right)^{2} \operatorname{Re}\left[\gamma_{c}(t)+\gamma_{p}(t)\right] .
$$

We evaluate the generalized diffusion coefficients in the weak-coupling regime when the memory effects are important at the initial stage of the adparticle motion. In Fig. 4 we present the time dependence of $D(t)=$ $D_{i n}(t)+D_{c o h}(t)$ at different temperatures. One can draw some conclusions after observation of the plots.

First of all, we see a non-monotonic behavior of $D(t)$, and these oscillations become more pronounced when the system temperature decreases. At low temperatures thermal fluctuations of the lattice are very small. Therefore, the lattice distortion caused by interaction of the adsorbate with the phonon subsystem has no time to relax, and the initial profile of the lattice potential has no time to be restored after the particle passage. As a result, the effective barrier is higher than its adiabatic value, and the particle starts to oscillate being caged in the deformed potential well. Such a behavior of $D(t)$ is observed even at the temperatures comparable with $\hbar \omega_{\max } / k_{B}$

Secondly, at temperatures $k_{B} T / \hbar \omega_{\max }<0.5$ (which are not presented in Fig. 4) these oscillations persist on the time scales, which are by two orders of magnitude higher than the inverse Debye frequency. But even at higher temperatures there are evident memory effects. 


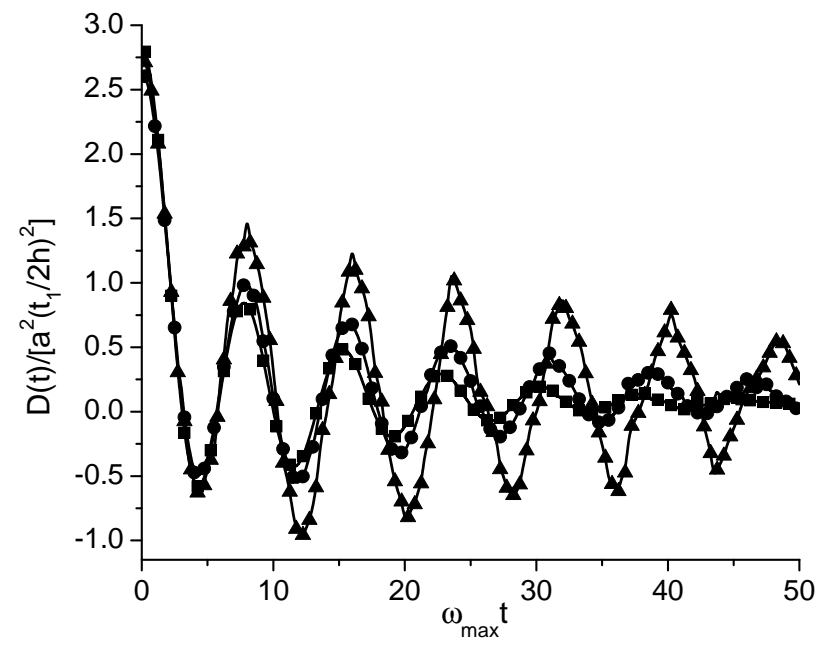

FIG. 4: Generalized diffusion coefficients calculated at $G=$ $10^{-3}, t_{1} / \hbar \omega_{\max }=10^{-5}, \Omega / \omega_{\max }=1$, and different temperatures: $k_{B} T / \hbar \omega_{\max }=0.5$ (triangles), 0.75 (circles) and 1 (squares).

In Ref. [26] we calculated the generalized diffusion coefficients at different values of $T$ and $\Omega$. The general tendencies observed in [26] can be formulated as follows: a low temperature $T$ and a high vibrational frequency $\Omega$ favor the oscillation dynamics of the particle, and so does a weak "substrate-adsorbate" interaction.

It would be interesting to relate this non-monotonic behavior of $D(t)$ to a possible recrossing phenomenon [7, 8, 25]. One can attribute the negative branches of $D(t)$ to the backward motion of the adsorbate: the particle may cross the dividing surface, located at the adsorption site $s$, due to the lattice distortion that "pushes" the particle in the opposite direction (with respect to that of the initial instant of motion). The nature of oscillations of $D(t)$ is different from that of $f_{s, s}(t)$, presented in Fig. 1. While in the coherent regime the only reason of the non-monotonic behavior is an interplay between the processes of transition and reflection of the wave packet, associated with the adparticle, the non-monotonic incoherent motion is determined by the adsorbate scattering on the substrate atoms (one can verify that only $D_{c o h}(t)$ contributes to the oscillatory adpartacle dynamics).

Now let us ask the question: what happens when one increases the value of coupling constant $G$ ? An intuitive answer would state that oscillations of $D(t)$ disappear at a moderate-to-strong coupling. Indeed, at strong coupling, when the energy exchange between the particle and the substrate atoms is faster, one can apply the Markovian approximation (4.4) for the coherent contribution to the generalized diffusion coefficient, which leads to the exponential relaxation of $D_{c o h}(t)$. However, fine features of such transition regimes, when the character of the adparticle motion changes from oscillatory to monotonic, need a thorough analysis in the framework of the non-Markovian approach.
Thus, if one increases the coupling constant $G$ until the oscillations of $D(t)$ disappear, one can obtain a critical value $G_{c r}(T, \Omega)$ as a function of the temperature and vibrational frequency, which separates two regimes of time evolution: there is a plain relaxation of $D(t)$ at $G>G_{c r}(T, \Omega)$, and a non-monotonic behavior at $G<G_{c r}(T, \Omega)$. A detailed analysis shows that, at first, the negative branch of $D(t)$ rises over the time axis, at that oscillations of the generalized diffusion coefficients still remain. So recrossing is vanishing, but the particle moves toward the nearest adsorption site at one moment faster, at another slower as if meeting obstacles.

At higher values of $G$ the oscillations completely disappear, and the adsorbate motion is governed mainly by the incoherent term $D_{\text {in }}(t)$. At $G \sim 0.1$ no coherent contribution is evident. Moreover, in the expression (6.2) for $D_{i n}(t)$ one has to use the strong-coupling limit for the end-preserving kernel (3.9) rather than the weak-coupling form (3.10). At such values of $G$ the endpreserving rate $\gamma_{p}(t)$ solely defines the adparticle dynamics, and the temperature dependence of the diffusion coefficient is given by Eq. (5.2).

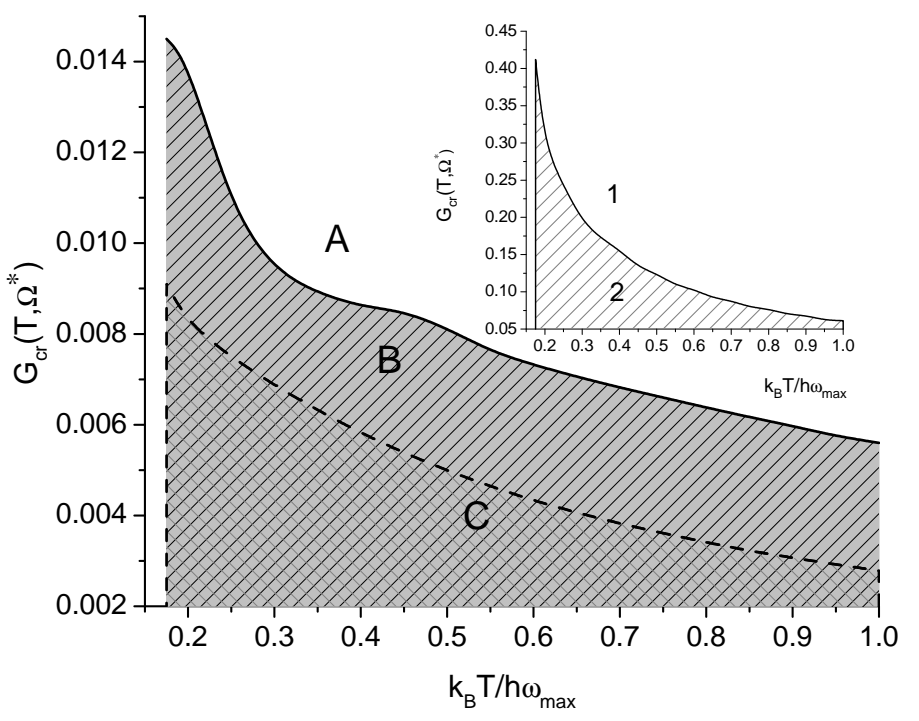

FIG. 5: The critical values of the coupling constants $G_{c r}\left(T, \Omega^{*}\right)$ at $\Omega^{*} / \omega_{\max }=1$, as functions of temperature $T$, that separate domains of the monotonic adparticle dynamics (A), oscillations without recrossing (B), and an eventual recrossing (C). The inset shows the temperature dependence of $G_{c r}\left(T, \Omega^{*}\right)$ that separates the strong-coupling (1) and weakcoupling (2) domains.

We present the above mentioned transition regimes of the adparticle motion in Fig. 5 and 6 as plots of $G_{c r}$ vs. temperature (at the fixed value of vibrational energy $\Omega^{*} / \omega_{\max }=1$, Fig. 5 ), and vs. vibrational energy (at the fixed value of temperature $k_{B} T / \hbar \omega_{\max }=0.5$, Fig. 6). When inspecting these curves, one can observe a remarkable feature: the transition domains of different dynamic regimes of $D(t)$ with respect to the coupling constant $G$ coincide with the regions, where the temperature be- 


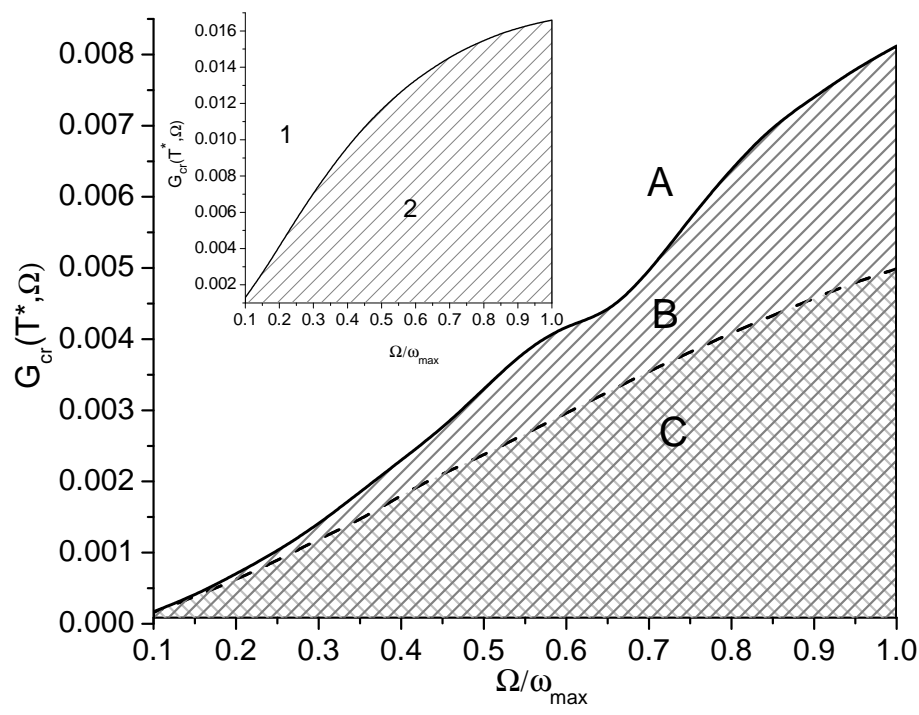

FIG. 6: The critical values of the coupling constants $G_{c r}\left(T^{*}, \Omega\right)$ at $k_{B} T^{*} / \hbar \omega_{\max }=0.5$, as functions of the vibrational frequency $\Omega$, that separate domains of the monotonic adparticle dynamics (A), oscillations without recrossing (B), and an eventual recrossing $(\mathrm{C})$. The inset shows the frequency dependence of $G_{c r}\left(T^{*}, \Omega\right)$ that separates domain (1), where $D_{\text {coh }}(t)$ decays as an exponential function (see Eq. (4.4) ), from that with essentially non-Markovian behavior of $D_{c o h}(t)$.

havior of the diffusion coefficients changes from a weakly dependent function of $T$ to quite a sensitive function of temperature. Namely, the region with a recrossing (the C-domain in Fig. 5) maps to a weakly dependent temperature regime (see Fig. 2), and the region with monotonic motion of the adparticle (the A-domain in Fig. 5) corresponds to a strongly dependent temperature regime (see Fig. 3). The domain B in Fig. 5, evidently, corresponds to transition of the temperature behavior of experimentally measured diffusion coefficient from weakly to strongly dependent function of $T$.

The same tendencies can be traced in Fig. 6, where the plot of $C_{c r}$ vs. $\Omega$ is presented at the fixed temperature. High values of the vibrational frequency extend the domains ( $\mathrm{B}$ and $\mathrm{C}$ ) of the non-monotonic adparticle dynamics. The insert in Fig. 6 shows that a transition from essentially non-Markovian dynamics to the case, when $D_{c o h}(t)$ decays as an exponential function (see Eq. (4.4)), occurs in a moderate-to-strong coupling domain. Of course, the most informative would be a threedimensional plot $G_{c r}(T, \Omega)$, but even such sections of the critical coupling constants at fixed temperature and vibrational energy, as in the above presented figures, give much food for thought.

One of the assumptions inferred can be formulated as follows. If the tendencies, presented in the "critical diagrams" in Figs. 5 and 6, remain in more sophisticated models describing quantum surface diffusion, and a correspondence between the "recrossing-monotonic motion" transition, and the change in the character of a
$T$-dependence of the experimentally measured diffusion coefficients $D_{\exp }$ is valid in general, then we could give a prognosis about the temperature behavior of $D_{\text {exp }}$, having only the information about the dynamics of the adsorbate at the initial stage of its motion. Otherwise, one has to measure the mean square displacement of the particle at times large enough to be sure that all remnants of the transition regimes are excluded. As it has been already mentioned, due to comparatively slow processes of quantum diffusion at low temperatures (where the aforesaid transition regimes are the most pronounced and durable), it would be useful to reduce the time of atom-tracking experiment, because the longer action of the measuring instrument, the greater influence on the system occurs.

It should be also noted that investigation of the transition regimes in the framework of two-level dissipative models is a topical problem [29], and much efforts are put into a study of the system crossover from one kind of its dynamics to another. In a recent paper 35] the authors obtained the "coherence-incoherence" transition diagrams for the dissipative two-level system with a nonzero bias and a sub-Ohmic bath as functions of the power index $n$ in a low-frequency asymptotics $J(\omega) \sim \omega^{n}$ of the spectral weight functions. Though their results were obtained in zero-temperature limit, it would be interesting to generalize such a model for $T \neq 0$ case to investigate whether there is any other reason (except the $G \rightarrow 0$ limit) for the "coherence-decoherence" transition.

At the end of the present Section we would like to touch upon the study of the recrossing phenomenon once more. Though the considered two-level dissipative model is too simplified to describe real "substrate-adsorbate" dynamics, especially in a comparison with the models traditionally used during a direct evaluation of the "fluxflux" time correlation functions [8, 25], it is worthy to compare them. On the one hand, in our model the lower temperature, the more pronounced oscillations of the generalized diffusion coefficient observed. This is different from the results of Ref. [8] where high $T$ favors the recrossing phenomenon. On the other hand, if we are in the low-temperature weak-coupling domain and increase the value of $G$, we will observe that there is a gradual transition from the domain $\mathrm{C}$ with a pronounced recrossing to the domain $\mathrm{B}$ (see Figs. 5 and 6 ) with oscillations but without recrossing. On the contrary, in the high-temperature region with $k_{B} T / \hbar \omega_{\max } \sim 1$ a gradual increase of the coupling constant will give us a sudden transition of the coherent part $D_{\text {coh }}(t)$ of the generalized diffusion coefficient from the domain $\mathrm{C}$ to the region $\mathrm{A}$ of the monotonic adparticle dynamics. However, this feature (not presented graphically because we investigate the crossover for the overall generalized diffusion coefficient $D(t)$ rather than for $\left.D_{c o h}(t)\right)$ is smeared out by the contribution of the incoherent term, which has a "long tail" behavior (3.10). If one could eliminate these long tails, introducing additional channels of the adparticle scattering, one would obtain an interesting result: 
though the recrossing is less pronounced at high $T$, it persists in a wider domain of coupling constant (the region B completely vanishes). Such a behavior would resemble the results of Ref. [8]. It has to be noted that the presented model in the general features corresponds to the model $\mathrm{F}$ of the cited paper (no surface motion is possible, both thermal fluctuations and lattice distortions are permitted).

\section{TIME DEPENDENCE OF THE TRANSITION PROBABILITIES $f_{s, s+n}(t)$}

In this section we study the off-diagonal nonequilibrium distribution functions $f_{s, s+n}(t)$. There are some reasons to look closer at the short-time dynamics of the above mentioned transition probabilities. First of all, the rate of the decay of $f_{s, s^{\prime}}(t)$ defines the time scales at which the contribution of $D_{c o h}(t)$ vanishes (let us remind that to derive the expression for the coherent term of the generalized diffusion coefficients we solved Eq. (3.2) with respect to $f_{s, s^{\prime}}(t)$, and inserted the obtained result in the upper equation (3.1)). On the other hand, the offdiagonal non-equilibrium distribution functions $f_{s, s+n}(t)$ can be related to the time dependent "flux-flux" crosscorrelation functions, determined at the adjacent sites $s$ and $s+n$. These time correlation functions are known to describe a multiple crossing or a multi-hopping regime [7, 8]. The multi-hopping facilitates an increase of the transport coefficients, in contrast to the recrossing, which reduces the total rate of the adparticle escape and lowers the value of the diffusion coefficient.

We evaluate the non-equilibrium transition probabilities $f_{s, s+n}(t)$, solving Eq. (3.1) with respect to the diagonal distribution functions $f_{s, s}(t)$ and inserting the obtained result in Eq. (3.2). The final expressions for the real and imaginary parts of $f_{s, s+n}(t)$ are

$\left.\operatorname{Re}\left[\tilde{f}_{s, s+n}(z)\right]=\frac{f_{s, s+n}(0)}{z+\operatorname{Re}\left[\tilde{\gamma}_{\text {inter }}(z)+\tilde{\gamma}_{\text {intra }}(z)-\tilde{\gamma}_{L L}^{+}(z)\right]}+o\left(t_{1}^{3} 0\right) .1\right)$

FIG. 7: Time dependence of the normalized function $\operatorname{Im}\left[P\left(r^{*}, t\right)\right] /\left[\operatorname{Im}\left[P\left(r^{*}, t\right)\right]\right]_{\max }$ at $r^{*}=r / a=1, G=10^{-3}$, $\Omega / \omega_{\max }=1, k_{B} T / \hbar \omega_{\max }=1$, and tunnelling amplitude Markovian and non-Markovian approximations.

where only linear terms in the tunnelling amplitude $t_{1}$ are retained. It has to be stressed that in the single particle limit there is no coupling between the real and imaginary parts, while in the general case of nonlinear kinetic equations $\operatorname{Re}\left[\tilde{f}_{s, s+n}(z)\right]$ and $\operatorname{Im}\left[\tilde{f}_{s, s+n}(z)\right]$ are coupled to each other. The second conclusion, which is to the point, is the following: the decay rate of $\operatorname{Re}\left[\tilde{f}_{s, s+n}(z)\right]$ is almost the same as that of the coherent part $D_{c o h}(t)$ of the generalized diffusion coefficient,which is defined by the kernel $\left.\tilde{\gamma}_{\text {total }}(z)=\tilde{\gamma}_{\text {inter }}(z)+\tilde{\gamma}_{\text {intra }}(z)+\tilde{\gamma}_{L L}^{+}(z)\right)$. Obviously, in zero-coupling limit Eq. (7.1) reproduces the value of $f_{s, s+n}(t)$ which does not depend on time in the coherent regime of motion, and the inverse Laplace transformation $t_{1} / \hbar \omega_{\max }=10^{-5}$. Solid and dashed lines correspond to the

of (7.2) in the limit $G \rightarrow 0$ gives us the expression (3.14) for $\operatorname{Im}\left[f_{s, s+n}(t)\right]$.

To study the time dependence of the transition probabilities we perform a certain simplification of Eq. (7.2). Namely, we pass from the difference form in the numerator of (7.2) to the derivative with respect to the adatom coordinate $r$. In fact, we perform the continuous media approximation, similarly like it was done in Ref. [26], when we obtained a Telegrapher's equation, describing the correlated random motion of the adparticle. After such a transformation, we are not bounded to a special geometry of the lattice any more and can rewrite Eq. (7.2) in the wave-vector-frequency representation for the imaginary part of the transition probability $\tilde{P}(k, z)$ as follows:

$$
\operatorname{Im}[\tilde{P}(k, z)]=\frac{\hbar k}{2 t_{\text {inter }}} \frac{\operatorname{Re}\left[\tilde{D}_{c o h}(z)\right]}{z+k^{2} \operatorname{Re}\left[\tilde{D}_{c o h}(z)+\tilde{D}_{i n}(z)\right]}(7
$$
tions, we can evaluate the $(r, t)$-dependence of the imaginary part of the transition probability. In Figs. 7 and 8

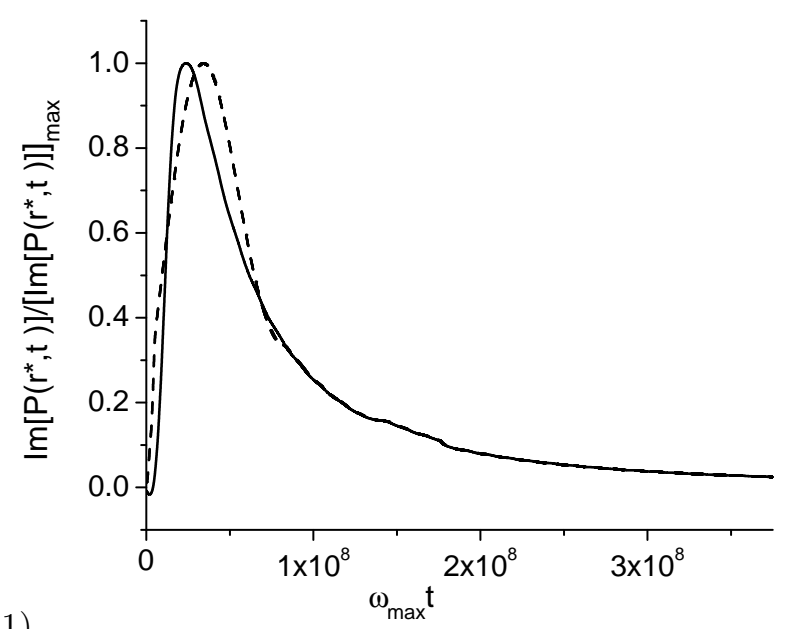

we present the time dependence of the above mentioned functions (normalized at their maximum values) at $r^{*}=$ $r / a=1$. The evaluation is performed for two different values of the tunnelling amplitude $t_{1} / \hbar \omega_{\max }=10^{-5}$ and $10^{-2}$. In the first case we observe that the adparticle reaches the point $r^{*}$ at times of about $10^{7}$ inverse Debye frequency. The adparticle motion is very slow, the lattice has enough time to relax at such huge times, and we do not observe any noticeable difference between the Markovian approximation for transition probability and the case, when the memory effects are taken into account.
Now, performing inverse Fourier and Laplace transforma- 


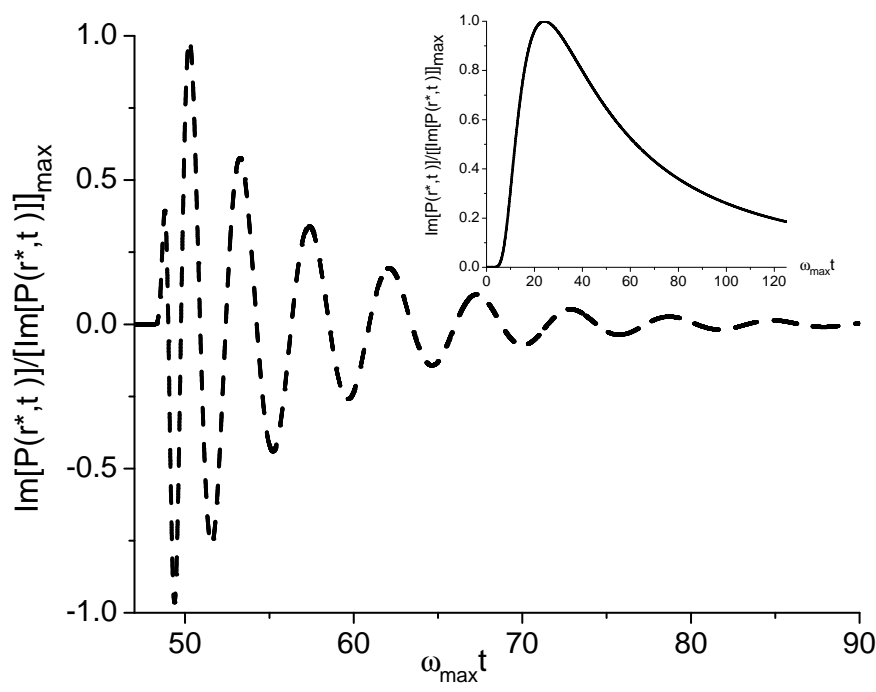

FIG. 8: Time dependence of the normalized function $\operatorname{Im}\left[P\left(r^{*}, t\right)\right] /\left[\operatorname{Im}\left[P\left(r^{*}, t\right)\right]\right]_{\max }$ at $r^{*}=r / a=1, G=10^{-3}$, $\Omega / \omega_{\max }=1, k_{B} T / \hbar \omega_{\max }=1$, and tunnelling amplitude $t_{1} / \hbar \omega_{\max }=10^{-2}$. Solid and dashed lines correspond to the Markovian and non-Markovian approximations.

A situation changes drastically (Fig. 8), when the value of the tunnelling amplitude increases by three orders of magnitude. The decay time of $P\left(r^{*}, t\right)$ is then comparable with that of the generalized diffusion coefficient (see Fig. 4 for comparison). The adparticle arrives at the point $r^{*}$ at times of about seven Debye periods $2 \pi / \omega_{\max }$, when the lattice has not relaxed completely, and the memory effects are still important. Thus, a transition state that originally obstructs a multi-hop from the site $s$ to the nearest neighboring one, is stabilized by the lattice fluctuations: the height of the effective barrier at $s+n$ can be lower than its adiabatic value at some instant of time, allowing the particle to perform a multiple crossing. We would like to note the strongly aperiodic behavior of the transition probability in contrast to the smooth relaxation in the Markovian case. The negative branches of $P\left(r^{*}, t\right)$ mean that the "transition window" for the multiple crossing is not always open, and at other instants of time the effective barrier height can exceed its static value, prohibiting multi-hops of the particle. On the other hand, the width of oscillations growths in time while their amplitude reduces. It means that multiple hops are less probable when time increases, but the period favorable for them to proceed becomes longer with increasing $t$.

We do not present plots for the real part of transition probability, because its time behavior does not differ from that of $D(t)$ (see Fig. 4). At the same time we have to emphasize that all the curves in Figs. 7 and 8 correspond to the normalized values of transition probabilities, while the non-normalized values in the continuous media limit would be much smaller than those of the distribution function $n\left(r^{*}, t\right)$ at the starting point $r^{*}=0$ of the adparticle motion. In fact, $n\left(r^{*}, t\right)$ tends to infinity at $r^{*}=0$, but even in the coherent limit and for a discrete lattice model the curve for $\operatorname{Im}\left[f_{0,1}(t)\right]$ lies much lower (see Fig. 1) than that for $f_{0,0}(t)$. It is obvious that the higher transition probability, the greater contribution of the multi-hops to the overall diffusion coefficient.

Thus, only rigorous evaluation of the site-dependent transition probabilities $f_{s, s+n}(t)$ for a lattice with given geometry would remove possible contradictions between the results, obtained for the real discrete structure of the surface, and our model case of a continuous media. Nevertheless, we believe that the basic conclusions drawn in this Section are quite reliable in the context of a qualitative analysis of the adparticle motion.

\section{CONCLUSIONS}

In this paper we made the systematic analysis of the dynamics of the adparticle, which performs an underbarrier tunnelling from one adsorption site to another as well as the vibrational transitions between two levels inside a quantum well, when its motion changes from coherent to incoherent due to interaction with acoustic surface phonons. We investigated the short-time dynamics of the particle in the case when its vibrational frequency $\Omega$ is comparable with the Debye frequency $\omega_{\max }$, and memory effects have to be taken into account. Though the latter are traditionally thought to be important only at transition regimes, when the adparticle motion is neither ballistic nor purely diffusive, and the experimentally measured diffusion coefficients do not have to remember the details of intermediate period, we showed that there is a close relation between the adparticle dynamics at times $\tau \sim 10 \omega_{\max }^{-1}$ and the temperature dependence of diffusion coefficients. Namely, as the coupling constant increases, the adparticle motion (initially oscillatory) becomes more and more smooth indicating that the temperature behavior of the diffusion coefficients $D(T)$ should change from weakly dependent on $T$ to quite a sensitive function of the temperature. Though we restricted ourselves by a comparatively simple two-level dissipative model, we believe that the above mentioned correspondence between the short-time dynamics of the adsorbate and the temperature behavior of the diffusion coefficients is valid for more sophisticated systems. If so, this " $T$ vs. $t$ " correspondence could be helpful at experimental evaluation of the diffusion coefficients because it would allow us to give a prognosis about their temperature behavior, having only an information about the adatom dynamics at the initial stage of its motion.

We showed that the coherent term of the generalized (time dependent) diffusion coefficient, which is defined by the adparticle scattering from the lattice, is responsible for the recrossing phenomenon at weak-coupling, but at high-coupling regime its contribution diminishes, and the particle motion is completely determined by the incoherent term. We performed a quantitative analysis of such transition regimes in terms of the critical coupling con- 
stant $G_{c r}(T, \Omega)$ which depends on the temperature and vibrational frequency. We compared the temperature dependence of the recrossing with the results obtained by a direct calculation of the "velocity-velocity" quantum time correlation functions [7, [] , and analyzed both common and different features of the adparticle dynamics.

While the generalized diffusion coefficients are connected to the "velocity-velocity" autocorrelation functions, the transition probabilities (non-diagonal distribution functions $\left.f_{s, s^{\prime}}(t)\right)$ can be related to the crosscorrelation function "velocity-velocity". A study of the transition probabilities is two-fold interesting: on the one hand, it shows how fast a loss of the adparticle coherence occurs; on the other hand, it allows to draw a conclusion about the phenomenon of multiple crossing. The contribution of multiple jumps to the diffusion coefficient is enhanced by the fact that in a double jump the random walker goes a double distance. Since the diffusion coefficients is defined by the squared jump length, the double jump can be important even if its jump rate is comparatively smaller than that of a single jump.

We investigated the time dependence of the transition probabilities in the model case of continuous media. It was shown that at very small values $t_{1} / \hbar \omega_{\max }=10^{-5}$ of the tunnelling amplitude the real part of the transition probability (which defines a multi-hop strength) decays at the same rate as the generalized diffusion coefficients, while the particle approaches the nearest adsorption site at much later times (which corresponds to the maximum value of the imaginary part of $\left.f_{s, s^{\prime}}(t)\right)$. The particle moves very slow, the lattice has plenty time to relax, and the Markovian approximation is quite applicable if one studies non-equilibrium transition probabilities. Contrary, at $t_{1} / \hbar \omega_{\max }=10^{-2}$ the typical times of decay for both real and imaginary parts of the transition probability are of the same order, and remnants of the memory effects have an influence on the multiple crossing, which is characterized by an aperiodic oscillatory function. We also verified that the zero-coupling limit reproduces the results for a coherent motion, when the strength of transition $\operatorname{Re}\left[f_{s, s+n}(t)\right]$ does not change in time, and $\operatorname{Im}\left[f_{s, s+n}(t)\right]$ is expressed analytically via Bessel functions.

The memory effects could be said to preserve a particle coherence to a certain extent: a portion of relaxation energy of the lattice is delivered to the adparticles preventing them from thermalization and maintaining the transition regimes from the coherent (ballistic) motion to the incoherent (diffusive) one. This reasoning would be even more realistic if one introduced the additional channels of the adparticle scattering: an electronic friction [14, 20], a non-linear "adsorbate-substrate" interaction [19] or a direct "adsorbate-adsorbate" interaction [21]. First of all, this would solve the "long-tails" problem of the kinetic kernels [22] and ensure their convergence without introduction of any additional parameter like a particle life-time $\omega_{0}^{-1}$ in a quantum well. On the other hand, additional interactions introduce new typical time scales, which could be well separated (the Markovian picture is then valid) or close to each other (then the nonMarkovian approach is necessary). Besides, by taking an "adsorbate-adsorbate" interaction into account one can go beyond the limits of small coverage and study a concentration dependence of the diffusion coefficients in addition to their temperature behavior [36]. We believe that all these directions are quite interesting from a viewpoint of the study of transition regimes of the adsorbate and could be the subject of future investigations.

\section{Acknowledgement}

This work was partially supported by the Project "Models of the quantum-statistical description of catalytic processes at the metallic surfaces" (Lviv Polytechnic National University), No. 0110U001091.

\section{Appendix}

To solve Eqs. 3.11-3.12) let us perform at first a Fourier transformation for the creation/annihilation operators

$$
a_{s}^{\dagger}=\frac{1}{\sqrt{N}} \sum_{k} \exp (i k s) a_{k}^{\dagger}, \quad a_{s}=\frac{1}{\sqrt{N}} \sum_{k} \exp (-i k s) a_{k}
$$

passing from the site representation $a_{s}^{\dagger}, a_{s}$ to wavevector representation $a_{k}^{\dagger}, a_{k}$ with $k=(2 \pi / N) m[k=$ $(2 \pi / N)(m+1 / 2)]$ for the lattice with the odd [even] numbers of adsorption sites and $m=-N / 2,-N / 2+$ $1, \ldots, N / 2-1$ [if $N$ is even] or $m=-(N-1) / 2,-(N-$ $1) / 2+1, \ldots,(N-1) / 2$ [if $N$ is odd].

Inserting A.1 in Eqs. (3.11)- (3.12) for the coherent motion, we obtain the following equation for the intermediate distribution function $F_{k, k^{\prime}}(t) \equiv\left\langle a_{k^{\prime}}^{\dagger} a_{k}\right\rangle^{t}$ :

$$
\frac{\partial F_{k, k^{\prime}}(t)}{\partial t}=-\frac{2 i t_{0}}{\hbar}\left(\cos k-\cos k^{\prime}\right) F_{k, k^{\prime}}(t),
$$

which is easily solved, giving

$$
F_{k, k^{\prime}}(t)=\exp \left[\frac{-2 i t_{0}}{\hbar}\left(\cos k-\cos k^{\prime}\right) t\right] .
$$

In the infinite lattice limit $N \rightarrow \infty$, we can pass from summation over $k$ to integration over continuous wavevector according to $\frac{1}{\sqrt{N}} \sum_{k} \cdots \longrightarrow \frac{1}{2 \pi} \int_{-\pi}^{\pi} \cdots d k$. After that we obtain an integral representation for the diagonal one-particle non-equilibrium distribution function as follows:

$$
\begin{aligned}
& f_{s, s}(\tau)=\frac{1}{4 \pi^{2}} \mid \int_{-\pi}^{\pi} d k[\cos (k s)+i \sin (k s)] \\
& \times\left.\exp \left[\frac{-2 i t_{0}}{\hbar} \tau \cos k\right]\right|^{2}=J_{s}^{2}\left(2 t_{0} \hbar^{-1} \tau\right),
\end{aligned}
$$


which is nothing but the squared s-th order Bessel function. Similarly, we can obtain the expression (3.14) for the imaginary part of the transition probability $f_{s, s+1}(t)$.

To show that the last term in Eq. (4.1) does not contribute to the mean square displacement $\left\langle\Delta r(t)^{2}\right\rangle=$ $a^{2} \sum_{s=1}^{N} s^{2} f_{s, s}(t)$ of the particle let us perform for simplicity the Markovian approximation for the generalized diffusion equation (4.1). Using Fourier transformation (A.1) we obtain the evolution equation for the intermediate distribution function in the following form:

$$
\begin{aligned}
& \frac{\partial F_{k k^{\prime}}(t)}{\partial t}=-\frac{F_{k k^{\prime}}(t)}{a^{2}}\left\{2\left[\tilde{D}_{c o h}(0)+\tilde{D}_{i n}(0)\right]\left[1-\cos \left(k-k^{\prime}\right)\right]\right. \\
& \left.+\tilde{D}_{c o h}(0)\left[\cos 2 k+\cos 2 k^{\prime}-2 \cos \left(k+k^{\prime}\right)\right]\right\}
\end{aligned}
$$

where the second term in the braces is related to the last term in the r.h.s of Eq. (4.1), which involves the "long distance" transition probabilities $f_{s \pm n, s \mp n}(t), f_{s, s \mp 2 n}(t)$.

The evolution equation for the mean square displacement can be written down as follows:

$$
\begin{aligned}
& \frac{d\left\langle\Delta r(t)^{2}\right\rangle}{d t}=a^{2} \sum_{s=1}^{N} \sum_{k, q} s^{2} \exp (i q s) \dot{F}_{k, k-q}(t) \\
& =-a^{2} \sum_{k, q} \dot{F}_{k, k-q}(t) \frac{d^{2}}{d q^{2}}\left(\sum_{s=1}^{N} \exp (i q s)\right) .
\end{aligned}
$$

Noting that the sum in the brackets yields $N$ times Kro- necker delta-symbol, which in the infinite lattice limit converts to Dirac delta-function $\delta(q)$, and integrating (A.6) by parts with taking into account (A.5), one can verify that only the first term in braces of Eq. A.5 contributes to the evolution equation for $\left\langle\Delta r(t)^{2}\right\rangle$ :

$$
\begin{aligned}
& \frac{d\left\langle\Delta r(t)^{2}\right\rangle}{d t}=\left[\tilde{D}_{c o h}(0)+\tilde{D}_{i n}(0)\right] / \pi \int_{-\pi}^{\pi} d k \\
& \left.\times \int_{-\pi}^{\pi} d q[1-\cos q] F_{k, k-q}(t) \frac{d^{2}}{d q^{2}} \delta(q)=2\left[\tilde{D}_{c o h}(0)+\tilde{D}_{i\left(\alpha_{2}(\mathrm{~A})\right.} \tilde{y}\right]\right)
\end{aligned}
$$

while the second term vanishes at the integration over $k$ :

$$
\begin{aligned}
& \int_{-\pi}^{\pi} d k \int_{-\pi}^{\pi} d q\{\cos (2 k)+\cos (k-q)-2 \cos (2 k-q)\} \\
& \times F_{k, k-q}(t) \frac{d^{2}}{d q^{2}} \delta(q)=\int_{-\pi}^{\pi} d k[2 \cos (2 k)-\cos k] F_{k, k}(t)(\mathrm{A} .8),
\end{aligned}
$$

because $F_{k, k}(t) \equiv 1$, and all derivatives of $F_{k, k}(t)$ with respect to wave-vector vanish.

The presented above calculation can be generalized to the $2 D$ case, or to the case when the memory effects are taken into account. As for the $2 D$ coherent regime, the only modification of Eqs. (3.13)-(3.14) consists in doubling of the power indexes at Bessel functions.
[1] Hydrogen Effects in Catalysis, edit. by Z. Paal and P. G. Menon Dekker, (New York, 1988).

[2] P. Costamagna, S. Srinivasan, Journ. Power Sourc. 102, 242 (2001).

[3] L.J. Lauhon and W. Ho, J. Phys. Chem. 105, 3987 (2000).

[4] I.C. da Cunha Lima, A. Troper, and S.C. Ying, Phys. Rev. B 41, 11798 (1990).

[5] V. Pouthier, J. C. Light, Journ. Chem. Phys. 133, 1204 (2000).

[6] T. Ala-Nissilayz, R. Ferrando, and S.C. Ying, Advances in Physics 51, No. 3, 949 (2002).

[7] G. Wahnström, K. Haug, and H. Metiu, Chem. Phys. Lett. 148, 158 (1988).

[8] T. Taniike, and K. Yamashita, Chem. Phys. 304, 159 (2004).

[9] W. Ho, Journ. Chem. Phys. 117, 11033 (2002).

[10] R. Baer, Y. Zeiri and R. Kosloff, Surf. Sci. 411, L783 (1998)

[11] S.C. Badescu, S.C. Ying, and T. Ala-Nissila, Phys. Rev. Lett. 86, 5092 (2001).

[12] P. Ohresser, H. Bulou, S.S. Dhesi, C. Boeglin, B. Lazarovits, E. Gaudry, I. Chado, J. Faerber, and F. Scheurer, Phys. Rev. Lett. 95, 195901 (2005).

[13] R. Martínez-Casado, A. Sanz, and S. Miret-Artés, J. Chem. Phys. 129, 184704 (2008).

[14] J. Kondo, Physica 125B, 279 (1984).
[15] R.F. Kiefl, R. Kadono, J.H. Brewer, G.M. Luke, H.K. Yen, M. Celio, and E.J. Ansaldo, Phys. Rev. Lett. 62, 792 (1989).

[16] Yu. Kagan, N.V. Prokofiev, Zh. Eksp. Teor. Fiz. 96, 2209 (1989) [Sov. Phys. JETP. 69, 1250 (1989)].

[17] P.D. Reilly, R.A. Harris, and K.B. Whaley, Journ. Chem. Phys. 95, 8599 (1991).

[18] P.D. Reilly, R.A. Harris, and K.B. Whaley, Journ. Chem. Phys. 97, 6975 (1992).

[19] X. D. Zhu and L. Deng, Phys. Rev. B 48, 17527 (1993).

[20] Yu. Kagan, N. V.Prokofiev, Zh. Eksp. Teor. Fiz. 90, 2176 (1986) [Sov. Phys. JETP. 63, 1276 (1986)].

[21] W. Brenig, Surf. Sci. 291, 207 (1993).

[22] D.N. Zubarev, V.G. Morozov, G. Röpke, Statistical Mechanics of Nonequilibrium Processes , (Fizmatlit, Moscow, 2002, in Russian).

[23] R. Ferrando, R. Spadacini, G.E. Tommei, and G. Caratti, Physica A 195, 506 (1993).

[24] R. Ferrando, R. Spadacini, G.E. Tommei, and G. Caratti, Surface Science, 311, 411 (1994).

[25] D.H. Zhang, J.C. Light, and Soo-Y. Lee, Journ. Chem. Phys. 111, 5741 (1999).

[26] V. V. Ignatyuk, Phys. Rev. E 80, 041133 (2009).

[27] V .V .Ignatyuk, V. G .Morozov, Condens. Matter Phys. 7, No 3(39), 579 (2004).

[28] P.D. Reilly, R.A. Harris, and K.B. Whaley, Phys. Rev. B 47, 5721 (1993). 
[29] A.J. Leggett, S. Chakravarty, A.T. Dorsey, M.P.A. Fisher, A. Garg, and W. Zwerger, Rev. Mod. Phys. 59, 1 (1987).

[30] I.M. Mryglod, I.P. Omelyan, and M.V. Tokarchuk, Mol. Phys. 84 235, (1995).

[31] V.V. Ignatyuk, I.M. Mryglod, and M.V. Tokarchuk, Low Temp. Phys. 25, 857 (1999).

[32] Z. Chvoj, J. Phys.: Cond. Matt. 12, 2135 (2000).

[33] D. Dou, J. Casas-Vazquez, and G. Lebon, Extended Irre- versible Thermodynamics (Springer, Berlin, 1998).

[34] R. Kubo, M. Toda and N. Hashitsume, Statistical Physics II (Springer, Berlin, 1991).

[35] Congjun Gan and Hang Zheng, Phys. Rev. E 80, 041106 (2009).

[36] A. Wong, A. Lee, and X.D. Zhu, Phys. Rev. B 51, 4418 (1995). 